\title{
A Coordinate Descent Framework to Joint Design of MPSK Sequences and Receive Filter Weights in MIMO Radar Systems
}

\author{
Mohammad Alaee-Kerahroodi ${ }^{\dagger *}$, Sadjad Imani ${ }^{\ddagger}$, Bhavani Shankar M. R..$^{\dagger}$, \\ Mohammad Mahdi Nayebi ${ }^{\ddagger}$, and Björn Ottersten ${ }^{\dagger}$ \\ ${ }^{\dagger}$ Interdisciplinary Centre for Security, Reliability and Trust (SnT), University of Luxembourg \\ $\ddagger$ Electrical Engineering Department, Sharif University, Tehran, Iran \\ *mohammad.alaee@uni.lu
}

\begin{abstract}
In this paper, we aim at a joint design of $M$ ary Phase Shift Keying (PSK) - or MPSK - transmit waveform and receive space-time filter to maximize the Signal to Interference plus Noise Ratio (SINR) in colocated Multiple Input Multiple Output (MIMO) radar systems. The design problem is formulated into a maximization of the SINR, including unimodular discrete phase constraint on the transmit waveforms. The resulting problem is non-convex, whereby we devise an iterative algorithm based on the Coordinate Descent (CD) framework to tackle it iteratively. To reduce the computational complexity in designing the transmit waveforms, we exploit the properties of Fast Fourier Transform (FFT) in this paper. Numerical examples illustrate the superior performance of the proposed algorithm in comparison with the state-ofthe-art counterparts.
\end{abstract}

Keywords - Coordinate Descent, Discrete Phase, Filter Optimization, MIMO Radar, Phased Array, Space Time Interference Suppression,

\section{Introduction}

Colocated MIMO radar systems, instead of spreading the radar geometry throughout the surveillance area, usually radiate orthogonal (or incoherent) waveforms their transmit antennas to enhance spatial resolution as well as improving detection performance, and refining parameter identifiability [1]. In a traditional Phased array system, additional antennas and related hardware are needed to improve spatial resolution. In a MIMO system, the transmitting signals from the single transmitters are different. As a result, the echo signals can be re-assigned to the source. This gives an enlarged virtual receive aperture. However, transmitting incoherent waveforms in these systems, leads to a loss in Signal to Noise Ratio (SNR) in comparison with the phased array counterpart [2].

To enhance SINR without losing the aforementioned advantages of the MIMO radar systems, a joint design of the transmit waveforms and the received filters is highlighted in the literature (see [3]-[16]). In [5], [6], [17], the phased-MIMO technique is proposed which exploit the benefits of both MIMO radar and phased array to suppress the energy received from the unwanted directions or sidelobe levels (SLLs). In this case, a scheme with different power transmitted from different antennas is needed, whereas due to the limitations of the Radio Frequency Power Amplifier (RFPA) ${ }^{1}$, these techniques increase the complexity and the cost of the whole system. A better solution is to design transmit covariance matrix (TCM) such that it enhances the detection performance, while transmitting identical power from each antenna element in the transmit array.

To generate and design TCM from the constant modulus waveforms, several approaches is proposed in the literature [4], [10], [14], [18]-[22]. In [10], a fixed rank-two positive semi-definite Toeplitz TCM is proposed for any number of transmitting antennas, which however can not exploit the full rank property of the MIMO radar systems in parameter identifiability and interference rejection. On the other hand, increasing the rank of covariance matrix, makes it difficult to extract actual waveforms ${ }^{2}$ whose covariance matrix is close to the designed version [23]. This will get worse, when number of transmit antennas [18], length of sequences [20], or alphabet ${ }^{3}$ size [19], [22], are small.

Recently, several papers focused on sequential optimization, to directly design transmit signal and the receive filter, achieving significant improvements in output SINR (see [9], [11], [13], and references therein). In [9], it is shown that MIMO radar systems can surpass the interferences with high level of accuracy and achieve high

1. RFPAs have nonlinear relationships between their input and output and they cannot have maximum power efficiency at all power levels [10].

2. Generally design of correlated waveforms for the desired transmit beampattern relies on a two step process: designing a covariance matrix of the waveforms; and synthesizing it to extract actual waveforms.

3. Radar engineers usually are interested to a family of phaseonly sequence which are chosen from a small size alphabet [24], [25]. Indeed, in order to control the spectrum sidelobe levels of the phase changing points, sets of sequences whose are chosen from a small size alphabet, are typically preferred [26]. 
output SINR in comparison with the phased array counterpart. Finally, in [13] transmit orthogonal waveforms are obtained by linearly combining a set of orthogonal sequences, achieving a comparable SINR with the nonorthogonal sequences.

In this paper, we aim at maximizing the SINR of a point-like target in the presence of signal dependent interferences, while imposing sets of $M$-ary PSK discrete phase sequences. By the method proposed in this paper, we fill the relevant gap of the design problem for SINR maximization constrained to the discrete phase alphabet. The non-convex constrained optimization problem is tackled using FFT, which makes it computationally attractive and easy to implement. We numerically show that the sets of $M$-ary PSK sequences that are designed with the algorithm proposed in this paper, have SINR neighboring to the one obtained from the nonconstrained optimization counterparts, indicating the superior performance of the proposed algorithms.

The rest of this paper is organized as follows. In Section 2 , SINR maximization problem related to the joint design of transmit sequence and receive filter is described. Then, the CD framework to tackle the non-convex optimization problem is obtained in Section 3. Section 4 provides several numerical experiments to verify the effectiveness of the proposed algorithm. Finally, Section 5 concludes the paper.

\section{System Model}

Consider a MIMO radar system with $N_{T}$ transmit antenna elements and $N_{R}$ receive antenna elements. Let

$$
\mathbf{s}(n)=\left[s_{1}(n), s_{2}(n), \ldots, s_{N_{T}}(n)\right]^{T} \in \mathbb{C}^{N_{T}},
$$

denotes the transmitted sequence at the $n$-th time sample. Assuming an Uniform Linear Array (ULA) with half-wavelength inter-element spacing for the transmit antenna elements, the complex baseband equivalent of the transmitted signals at target location $\theta=\theta_{0}$ can be expressed as

$$
\mathbf{a}_{T}^{H}\left(\theta_{0}\right) \mathbf{s}(n), n=1, \ldots, N
$$

where $N$ is the sequence length and

$$
\mathbf{a}_{T}(\theta)=\left[1, e^{-\jmath \pi \sin (\theta)}, \ldots, e^{-\jmath \pi\left(N_{T}-1\right) \sin (\theta)}\right]^{T} \in \mathbb{C}^{N_{T}} .
$$

Suppose there are $Q$ signal dependent interference sources located at the look directions $\theta \neq \theta_{0}, j=$ $1,2, \ldots, Q$. The baseband equivalent of the received signals are given by,

$$
\begin{aligned}
\mathbf{y}(n)=\alpha_{0} \mathbf{a}_{R}\left(\theta_{0}\right) \mathbf{a}_{T}^{H}\left(\theta_{0}\right) \mathbf{s}(n) & \\
& +\sum_{j=1}^{Q} \alpha_{j} \mathbf{a}_{R}\left(\theta_{j}\right) \mathbf{a}_{T}^{H}\left(\theta_{j}\right) \mathbf{s}(n)+\mathbf{n}(n)
\end{aligned}
$$

which is reflected from a point-like target ${ }^{4}$ located at look angle $\theta=\theta_{0}$, contaminated with white zero mean

4. A target within a range cell.
Gaussian noise $\mathbf{n}(n) \in \mathbb{C}^{N_{R}}$ with covariance matrix $\sigma_{n}^{2} \mathbf{I}$, and $Q$ signal dependent interfering sources at $\theta=\theta_{j}$. Above, $\mathbf{a}_{R}(\theta)$ is the receive steering vector, which is similarly defined as $\mathbf{a}_{T}(\theta)$, precisely

$$
\mathbf{a}_{R}(\theta)=\left[1, e^{-\jmath \pi \sin (\theta)}, \ldots, e^{-\jmath \pi\left(N_{R}-1\right) \sin (\theta)}\right]^{T} \in \mathbb{C}^{N_{R}} .
$$

Also, $\alpha_{0}$ and $\alpha_{j}$, are unknown deterministic parameters accounting for both reflectivity and channel propagation effects of the target and the $j$-th interference source, respectively. Further, collecting all the subpulses, the received signal in matrix form can be written as,

$$
\mathbf{Y}=\alpha_{0} \mathbf{a}_{R}\left(\theta_{0}\right) \mathbf{a}_{T}\left(\theta_{0}\right)^{H} \mathbf{S}+\sum_{j=1}^{Q} \alpha_{j} \mathbf{a}_{R}\left(\theta_{j}\right) \mathbf{a}_{T}\left(\theta_{j}\right)^{H} \mathbf{S}+\mathbf{V}
$$

where $\mathbf{Y}=[\mathbf{y}(1), \mathbf{y}(2), \ldots, \mathbf{y}(N)] \in \mathbb{C}^{N_{R} \times N}$,

$$
\begin{aligned}
\mathbf{S} & =[\mathbf{s}(1), \mathbf{s}(2), \ldots, \mathbf{s}(N)] \in \mathbb{C}^{N_{T} \times N}, \\
\mathbf{V} & =[\mathbf{n}(1), \mathbf{n}(2), \ldots, \mathbf{n}(N)] \in \mathbb{C}^{N_{R} \times N} .
\end{aligned}
$$

The received signal is then vectorized as,

$$
\mathbf{z}=\alpha_{0} \mathbf{A}\left(\theta_{0}\right) \mathbf{x}+\sum_{j=1}^{Q} \alpha_{j} \mathbf{A}\left(\theta_{i}\right) \mathbf{x}+\mathbf{v}
$$

where $\mathbf{z}=\operatorname{vec}(\mathbf{Y}) \in \mathbb{C}^{N N_{R}}, \mathbf{x}=\operatorname{vec}(\mathbf{S}) \in \mathbb{C}^{N N_{T}}$ and $\mathbf{v}=\operatorname{vec}(\mathbf{V}) \in \mathbb{C}^{N N_{R}}$, with

$$
\mathbf{A}(\theta)=\mathbf{I}_{N} \otimes\left[\mathbf{a}_{R}(\theta) \mathbf{a}_{T}(\theta)^{H}\right],
$$

which is a direct result of $\operatorname{vec}(\mathbf{A B C})=$ $(\mathbf{I} \otimes \mathbf{A C}) \operatorname{vec}(\mathbf{B})$. Also, $\mathbf{v}$ is the noise vector with zero mean and covariance matrix $\sigma_{n}^{2} \mathbf{I}_{N N_{R}}$. The vector of observation $\mathbf{z}$ is filtered ${ }^{5}$ through the weight vector $\mathbf{w}$, i.e., $\mathbf{r}=\mathbf{w}^{H} \mathbf{z}$, and then the output SINR can be expressed as

$$
\operatorname{SINR} \equiv f(\mathbf{x}, \mathbf{w})=\frac{\sigma_{0}^{2}\left|\mathbf{w}^{H} \mathbf{A}\left(\theta_{0}\right) \mathbf{x}\right|^{2}}{\mathbf{w}^{H} \mathbf{R}_{c}(\mathbf{x}) \mathbf{w}+\mathbf{w}^{H} \mathbf{w} \sigma_{n}^{2}},
$$

with $\mathbf{R}_{c}(\mathbf{x})=\sum_{j=1}^{Q} \sigma_{j}^{2} \mathbf{A}\left(\theta_{j}\right) \mathbf{x x}^{H} \mathbf{A}\left(\theta_{j}\right)^{H}$. In the following, we aim to jointly design the transmit waveform $\mathbf{x}$ and the receive space-time filter $\mathbf{w}$ in order to maximize SINR given knowledge of the interferences (locations and strengths). This is equivalent to the following optimization problem:

$$
\mathcal{P}_{\mathbf{x}, \mathbf{w}}^{L}= \begin{cases}\max _{\mathbf{x}, \mathbf{w}} & f(\mathbf{x}, \mathbf{w}) \\ \text { s.t. } & \mathbf{x} \in \Omega_{L}\end{cases}
$$

where the constraint $\mathbf{x} \in \Omega_{L}$ identify discrete phase, where the feasible set is restricted to a finite number of equi-spaced points on the unit circle. Precisely,

$$
\Omega_{L}=\left\{\mathbf{x} \mid x_{n} \in \Psi_{L}, n=1, \ldots, N N_{T}\right\},
$$

where $\Psi_{L}=\left\{1, \bar{\omega}, \ldots, \bar{\omega}^{L-1}\right\}, \bar{\omega}=e^{\jmath \frac{2 \pi}{L}}$ and $L$ is the size of discrete constellation alphabet.

5. The receive filter $\mathbf{w}$, is also referred to as the receive beamformer. 


\section{Joint Design of the Receive Space- Time Filter and the Transmit Sequences}

In this section, we deal with the joint design of the radar code and the corresponding receive space-time filter under practical constraint on the phase of the transmit code sequences. Notice that the aforementioned optimization problem is non-convex, multi-variable, and constrained. A viable means to handle these type of problems, is to use the alternative optimization [8], [12], by iteratively tackling the original problem.

\subsection{Optimum Space-Time Receive Filter}

Let us assume $\mathbf{x}^{(k)}$ is an admissible radar code at iteration $(k)$ for the Problem $\mathcal{P}_{\mathbf{x}, \mathbf{w}}^{L}$. The optimal spacetime receive filter $\mathbf{w}^{(k)}$ can be obtained by solving the following optimization problem

$$
\max _{\mathbf{w}} \frac{\sigma_{0}^{2}\left|\mathbf{w}^{H} \mathbf{A}\left(\theta_{0}\right) \mathbf{x}^{(k)}\right|^{2}}{\mathbf{w}^{H} \mathbf{R}_{c}\left(\mathbf{x}^{(k)}\right) \mathbf{w}+\mathbf{w}^{H} \mathbf{w} \sigma_{n}^{2}} .
$$

Notice that, (10) is the well-known Minimum Variance Distortionless Response (MVDR) problem [27], and its solution can be obtained as,

$$
\mathbf{w}^{(k)}=\left(\mathbf{R}_{c}\left(\mathbf{x}^{(k)}\right)+\sigma_{n}^{2} \mathbf{I}\right)^{-1} \mathbf{A}\left(\theta_{0}\right) \mathbf{x}^{(k)} .
$$

\subsection{Optimum Transmit Sequences}

To obtain the optimized code vector $\mathbf{x}^{(k)}$, we use CD approach to sequentially optimize the objective over one variable keeping fixed the others. Indeed, starting from a receive filter $\mathbf{w}^{(k-1)}$, we search for an admissible radar code entry $x_{d}$ at iteration $(k)$, tackling

$$
\begin{cases}\max _{x_{d}} & \frac{\sigma_{0}^{2}\left|\mathbf{w}^{(k-1)^{H}} \mathbf{A}\left(\theta_{0}\right) \widetilde{\mathbf{x}}\right|^{2}}{\mathbf{w}^{(k-1)}{ }^{H} \mathbf{R}_{c}(\widetilde{\mathbf{x}}) \mathbf{w}^{(k-1)}+\mathbf{w}^{(k-1)^{H}} \mathbf{w}^{(k-1)} \sigma_{n}^{2}}, \\ \text { s.t. } & x_{d} \in \Omega_{L}\end{cases}
$$

where

$$
\widetilde{\mathbf{x}}=\left[x_{1}^{(k-1)}, \ldots, x_{d-1}^{(k-1)}, x_{d}, x_{d+1}^{(k-1)}, \ldots, x_{N}^{(k-1)}\right]^{T} \in \mathbb{C}^{N} .
$$

Whenever $x_{d}^{\star}$ is found, we update $\mathbf{x}^{(k)}=\widetilde{\mathbf{x}}^{\star}$ where

$$
\widetilde{\mathbf{x}}^{\star}=\left[x_{1}^{(k-1)}, \ldots, x_{d-1}^{(k-1)}, x_{d}^{\star}, x_{d+1}^{(k-1)}, \ldots, x_{N}^{(k-1)}\right]^{T} \in \mathbb{C}^{N} .
$$

Note that the other entries other than $x_{d}$ are retained from the previous iteration. Let us assume $x_{d}$ is the only variable of the code vector $\mathbf{x}$, and $\mathbf{x}_{-d}^{(k)}$ refers to the remaining code entries which are assumed to be known and fixed at iteration $(k+1)$. We define

$$
f_{\mathbf{w}}\left(x_{d}, \mathbf{x}_{-d}^{(k)}\right) \equiv \frac{\sigma_{0}^{2}\left|\mathbf{w}^{(k)^{H}} \mathbf{A}\left(\theta_{0}\right) \widetilde{\mathbf{x}}\right|^{2}}{\mathbf{w}^{(k)}{ }^{H} \mathbf{R}_{c}(\widetilde{\mathbf{x}}) \mathbf{w}^{(k)}+\mathbf{w}^{(k)^{H}} \mathbf{w}^{(k)} \sigma_{n}^{2}},
$$

where

$$
\widetilde{\mathbf{x}}_{-d}^{(k)}=\left[x_{1}^{(k)}, \ldots, x_{d-1}^{(k)}, x_{d+1}^{(k)}, \ldots, x_{N}^{(k)}\right]^{T} \in \mathbb{C}^{N-1},
$$

is known from $k$-th iteration. Thus, the non-convex constrained optimization problem $(12)$ at iteration $(k+1)$ can be recast as,

$$
\overline{\mathcal{P}}_{d, \mathbf{x}^{(k)}}^{L}=\left\{\begin{array}{ll}
\max _{x_{d}} & f_{\mathbf{w}}\left(x_{d}, \mathbf{x}_{-d}^{(k)}\right) \\
\text { s.t. } & x_{d} \in \Omega_{L}
\end{array} .\right.
$$

To tackle $\overline{\mathcal{P}}_{d, \mathbf{x}^{(k)}}^{L}$, first notice that the numerator of $f_{\mathbf{w}}\left(x_{d}, \mathbf{x}_{-d}^{(k)}\right)$ can be written as

$$
\begin{aligned}
\sigma_{0}^{2} \mid & \left.\mathbf{w}^{(k)^{H}} \mathbf{A}\left(\theta_{0}\right) \widetilde{\mathbf{x}}\right|^{2} \\
& =\sigma_{0}^{2} \operatorname{tr}\left(\mathbf{w}^{(k)^{H}} \mathbf{A}\left(\theta_{0}\right) \widetilde{\mathbf{x}} \widetilde{\mathbf{x}}^{H} \mathbf{A}\left(\theta_{0}\right)^{H} \mathbf{w}^{(k)}\right) \\
& =\sigma_{0}^{2} \operatorname{tr}\left(\widetilde{\mathbf{x}}^{H} \mathbf{A}\left(\theta_{0}\right)^{H} \mathbf{w}^{(k)} \mathbf{w}^{(k)^{H}} \mathbf{A}\left(\theta_{0}\right) \widetilde{\mathbf{x}}\right) \\
& =\sigma_{0}^{2} \widetilde{\mathbf{x}}^{H} \mathbf{B}_{\theta_{0}, \mathbf{w}} \widetilde{\mathbf{x}},
\end{aligned}
$$

where $^{6} \mathbf{B}_{\theta_{0}, \mathbf{w}}=\mathbf{A}\left(\theta_{0}\right)^{H} \mathbf{w}^{(k)} \mathbf{w}^{(k)}{ }^{H} \mathbf{A}\left(\theta_{0}\right)$. Noticing to the phase only constraints on $x_{d}$, we use the change of variable $x_{d}=e^{\jmath \phi_{d}}$ and resort to ${ }^{7}$

$$
f_{\mathbf{w}}\left(\phi_{d}, \mathbf{x}_{-d}^{(k)}\right)=\sigma_{0}^{2} \frac{\eta_{\mathbf{w}}\left(\phi_{d}, \mathbf{x}_{-d}^{(k)}\right)}{\lambda_{\mathbf{w}}\left(\phi_{d}, \mathbf{x}_{-d}^{(k)}\right)+\sigma_{n}^{2}},
$$

as the objective function, where by expanding (14) and collecting terms dependent on $\phi_{d}$ and those independent of them, we get

$$
\eta_{\mathbf{w}}\left(\phi_{d}, \mathbf{x}_{-d}^{(k)}\right)=a_{1}^{(k)}+a_{2}^{(k)} e^{-\jmath \phi_{d}}+a_{3}^{(k)} e^{\jmath \phi_{d}}
$$

with

$$
\begin{gathered}
a_{1}^{(k)}=\sum_{\substack{t=1 \\
t \neq d}}^{N N_{T}} \sum_{\substack{q=1 \\
q \neq d}}^{N N_{T}} \mathbf{B}_{\theta_{0}, \mathbf{w}}(t, q) x_{q}^{(k)} x_{t}^{(k)^{*}}+\mathbf{B}_{\theta_{0}, \mathbf{w}}(d, d), \\
a_{2}^{(k)}=\sum_{\substack{t=1 \\
t \neq d}}^{N N_{T}} \mathbf{B}_{\theta_{0}, \mathbf{w}}(t, d) x_{t}^{(k)^{*}}, \\
a_{3}^{(k)}=\sum_{\substack{q=1 \\
q \neq d}}^{N N_{T}} \mathbf{B}_{\theta_{0}, \mathbf{w}}(d, q) x_{q}^{(k)} .
\end{gathered}
$$

Similarly, expanding the denominator of (13) into terms dependent and independent of $\phi_{d}$, we have,

$$
\lambda_{\mathbf{w}}\left(\phi_{d}, \mathbf{x}_{-d}^{(k)}\right)=b_{1}^{(k)}+b_{2}^{(k)} e^{-\jmath \phi_{d}}+b_{3}^{(k)} e^{\jmath \phi_{d}},
$$

with

$$
b_{1}^{(k)}=\sum_{\substack{t=1 \\ t \neq d}}^{N N_{T}} \sum_{\substack{q=1 \\ q \neq d}}^{N N_{T}} \mathbf{B}_{\theta_{I}, \mathbf{w}}(t, q) x_{q}^{(k)} x_{t}^{(k)^{*}}+\mathbf{B}_{\theta_{I}, \mathbf{w}}(d, d),
$$

6. The dependence of $\mathbf{B}_{\theta_{0}, \mathbf{w}}$ to iteration $(k+1)$ is explicit to ease the notation.

7. We assume to have normalized receive space time filter (i. e., $\left.\mathbf{w}^{(k){ }^{H}} \mathbf{w}^{(k)}=1\right)$. 


$$
\begin{aligned}
b_{2}^{(k)} & =\sum_{\substack{t=1 \\
t \neq d}}^{N N_{T}} \mathbf{B}_{\theta_{I}, \mathbf{w}}(t, d) x_{t}^{(k)^{*}}, \\
b_{3}^{(k)} & =\sum_{\substack{q=1 \\
q \neq d}}^{N N_{T}} \mathbf{B}_{\theta_{I}, \mathbf{w}}(d, q) x_{q}^{(k)} .
\end{aligned}
$$

where $\mathbf{B}_{\theta_{I}, \mathbf{w}}=\sum_{j=1}^{Q} \sigma_{j}^{2} \mathbf{A}\left(\theta_{j}\right) \mathbf{w}^{(k)} \mathbf{w}^{(k)^{H}} \mathbf{A}\left(\theta_{j}\right)^{H}$. Consequently, the optimization problem with explicit dependence to $\phi_{d}$ can be written as

$$
\overline{\mathcal{P}}_{d, \phi_{d}(k)}^{L}= \begin{cases}\max _{\phi_{d}} & \sigma_{0}^{2} \frac{\eta_{\mathbf{w}}\left(\phi_{d}, \mathbf{x}_{-d}^{(k)}\right)}{\lambda_{\mathbf{w}}\left(\phi_{d}, \mathbf{x}_{-d}^{(k)}\right)+\sigma_{n}^{2}} \\ \text { s.t. } & \phi_{d} \in\left\{0, \frac{2 \pi}{L}, \frac{4 \pi}{L}, \ldots, \frac{2 \pi(L-1)}{L}\right\}\end{cases}
$$

the following lemma provides a key result to tackle Problem $\overline{\mathcal{P}}_{d, \phi_{d}(k)}^{L}$.

Lemma 3.1. Let $\boldsymbol{\rho}\left(\theta_{l}\right)=\left|\alpha+\beta e^{-\jmath \theta_{l}}+\gamma e^{\jmath \theta_{l}}\right|$, with $\theta_{l}=\frac{2 \pi(l-1)}{L}, l=1, \ldots, L$, and $\boldsymbol{\rho}(\theta) \in \mathcal{R}^{L}$, then

$$
\boldsymbol{\rho}(\theta)=\left|D F T\left[\gamma, \beta, \alpha, \mathbf{0}_{1 \times(L-3)}\right]^{T}\right| .
$$

Proof. Proof is straight forward and can be obtained with the insight of Appendix $\mathrm{C}$ in [24].

Inspired from Lemma 3.1, we can efficiently calculate $\eta_{\mathbf{w}}\left(\phi_{d}, \mathbf{x}_{-d}^{(k)}\right)$ and $\lambda_{\mathbf{w}}\left(\phi_{d}, \mathbf{x}_{-d}^{(k)}\right)$ using the FFT. Putting them into the $\overline{\mathcal{P}}_{d, \phi_{d}(k)}^{L}$, we obtain

$$
\phi_{d}^{\star}=\frac{2 \pi\left(l^{\star}-1\right)}{L},
$$

with

$$
l^{\star}=\arg \max \frac{\sigma_{0}^{2}\left|a_{1}^{(k)}+a_{2}^{(k)} e^{-\jmath \theta_{l}}+a_{3}^{(k)} e^{\jmath \theta_{l}}\right|}{\left|b_{1}^{(k)}+b_{2}^{(k)} e^{-\jmath \theta_{l}}+b_{3}^{(k)} e^{\jmath \theta_{l}}\right|+\sigma_{n}^{2}} .
$$

Finlay, optimal code entry can be calculate with

$$
x_{d}^{\star}=e^{\jmath \phi_{d}^{\star}} .
$$

Notice that, the monotonic property of the CD technique along with the fact that the objective function is bounded (from top) are sufficient to prove the convergence of the sequence of objective values.

A summary of the proposed approach can be found in Algorithm 1.

\section{Performance Analysis}

In this section, several numerical examples are provided to evaluate the performance of the proposed algorithms in term of SINR, convergence and interference suppression. In all of the examples, we assume transmit and receive array exploits a ULA with half element spacing, where number of sub-pulses in each transmit
Algorithm 1 Joint Design of MPSK Sequences and Receive Filter Weights.

Input: Initial code $\mathbf{x}_{0} \in \Omega_{L}$, space-time filter coefficient $\mathbf{w}_{0}, \theta_{0}, \theta_{j}$, and stopping threshold $\tau$;

Output: Optimal solution $\mathbf{x}^{\star}, \mathbf{w}^{\star}$;

\section{1) Initialization.}

- Compute the initial objective value $f\left(\mathbf{x}_{0}, \mathbf{w}_{0}\right)$ using equation (9);

- $\quad$ Set $k, d=1$;

2) Improvement.

- do

a) Solve (12) and obtain the optimal code entry $x_{d}^{\star}$;

b) $\mathbf{x}^{(k)}=\left[x_{1}^{(k-1)}, \ldots, x_{d}^{\star}, \ldots, x_{N}^{(k-1)}\right]^{T}$;

c) update $\mathbf{w}^{(k)}=$ $\left(\mathbf{R}_{c}\left(\mathbf{x}^{(k)}\right)+\sigma_{n}^{2} \mathbf{I}\right)^{-1} \mathbf{A}\left(\theta_{0}\right) \mathbf{x}^{(k)} ;$

d) $\quad$ set $d:=d+1, k:=k+1$;

- until $d>N$;

3) Stopping Criterion.

- If $\left|f\left(\mathbf{x}^{(k)}, \mathbf{w}^{(k)}\right)-f\left(\mathbf{x}^{(k-N)}, \mathbf{w}^{(k-N)}\right)\right|<$ $\tau$, stop. Otherwise, set $d:=1$ and to the step 2);

4) Output.

- $\quad$ Set $\mathbf{x}^{\star}=\mathbf{x}^{(k)}, \mathbf{w}^{\star}=\mathbf{w}^{(k)}$.

signal is $N=16$, and the stop criterion is $\tau=10^{-3}$. Also, total transmit power is $1, \sigma_{n}^{2}=1$. Consequently, the SNR and Interfernece to Noise Ratio (INR) are given by, SNR $=10 \log 10 \frac{\mathrm{E}\left\{\left|\alpha_{0}\right|^{2}\right\}}{\sigma_{n}^{2}}, \mathrm{INR}_{j}=10 \log 10 \frac{\mathrm{E}\left\{\left|\alpha_{j}\right|^{2}\right\}}{\sigma_{n}^{2}}$. The method in [10], [13] are chosen as the benchmarks and the conventional phased array with similar physical array as to the MIMO, equipped with MVDR beamformer is adopted for the comparison purposes.

First simulation depicts the convergence behavior of the proposed algorithm. We assume $N_{T}=N_{R}=4$, a target is located at $\theta_{0}=10^{\circ}$ with $\mathrm{SNR}=20 \mathrm{~dB}$, and also two signal dependent interferences are located at $\theta_{1}=$ $-25^{\circ}$ and $\theta_{2}=40^{\circ}$ with INR $=30 \mathrm{~dB}$. Discrete phases are obtained when $L=4$ and $L=16$ which is based on FFT implementation. As we can see in Figure 1, the proposed algorithm monotonically increases the SINR values. The upper bound for SINR improvement is equal to the SNR of the targets, when there is no interference, and the received signal is only contaminated with the thermal noise of the receivers.

The SINR values obtained via the proposed algorithm in comparison with the benchmarks and phased array radar system is depicted in Figure 2. We assume that a target is located at $\theta_{0}=0^{\circ}$ with $\mathrm{SNR}=20 \mathrm{~dB}$, when 6 signal dependent interferences are located at 


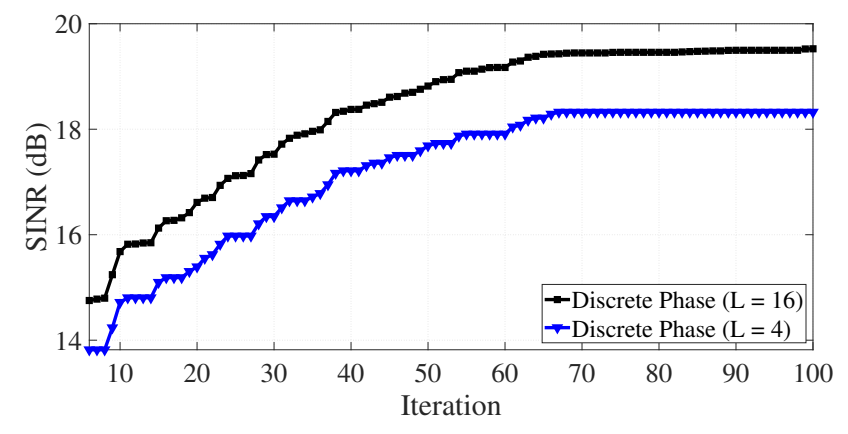

Figure 1. Convergence behavior of the proposed algorithms when $N_{T}=N_{R}=4, \theta_{0}=10, \mathrm{SNR}=20 \mathrm{~dB}, \theta_{1}=-25^{\circ}, \theta_{2}=40^{\circ}$, $\mathrm{INR}=30 \mathrm{~dB}, N=16$ and $L=4,16$.

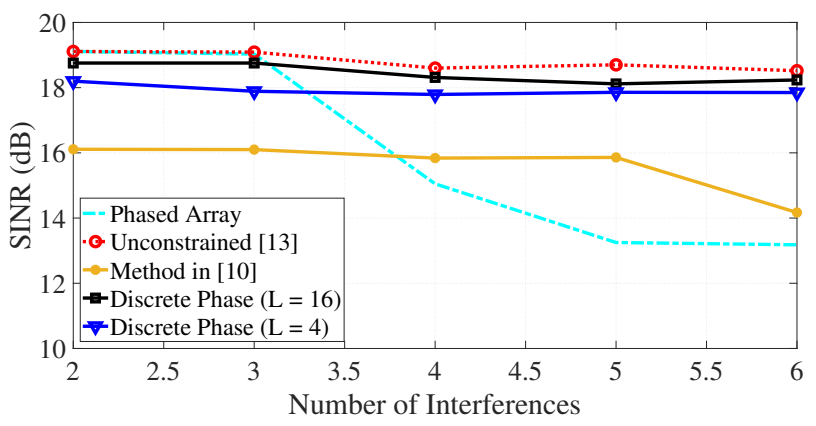

Figure 2. Comparison of degrees of freedom in interference suppression for proposed Continuous and discrete phase algorithm with phased-array, unconstrained MIMO radar in [13] and [10], $N_{T}=N_{R}=4, \theta_{0}=0, \mathrm{SNR}=20 \mathrm{~dB}, \theta_{j}=-19^{\circ}, 31^{\circ}, 44^{\circ},-38^{\circ}$, $59^{\circ},-64^{\circ}, \mathrm{INR}=30 \mathrm{~dB}, N=16$ and $L=4,16$.

$-19^{\circ}, 31^{\circ}, 44^{\circ},-38^{\circ}, 59^{\circ}$ and $-64^{\circ}$ with INR $=30 \mathrm{~dB}$. This figure shows that the SINR values obtained via the proposed method $(L=4,16)$ is neighboring to the unconstrained method in [13], indicating the superiority of the proposed algorithm. Also, with the proposed method, the SINR values are kept almost fixed while the number of interference is increasing. Notice that, the rank of correlated MIMO radar in [10] is always 2 , and that's why this method is not able to use the whole waveform diversity superiority of the MIMO radar systems. Also, the method in [10] is not a joint transmit receive design and therefore, even with small number of interferences its performance is less than the others. On the other hand, the performance of the phased array radar decreases when the number of interferences is greater than $N_{R}-1$.

Figure 3 , shows the real and imaginary parts of the obtained sequence from the scenario investigated for Figure 2. A comparison between Figure 3 and Figure 2 indicates that, the novel algorithm proposed in this paper has the advantage of designing $M$-ary PSK sequences with high SINR, but small alphabet size in the same time.

Eventually, the joint transmit and receive power pattern (transceiver pattern) of the obtained $M$-ary

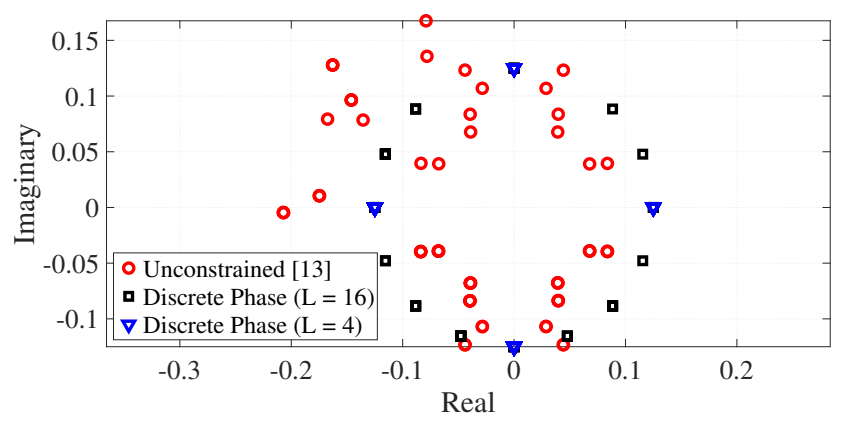

Figure 3. comparison of Real and Imaginary parts of transmit signal obtained using proposed Continuous and discrete phase algorithm and unconstrained MIMO radar in [13]. $N_{T}=N_{R}=4$, $\theta_{0}=10, \mathrm{SNR}=20 \mathrm{~dB}, \theta_{1}=-25^{\circ}, \theta_{2}=40^{\circ}, \mathrm{INR}=30 \mathrm{~dB}, N=16$ and $L=4,16$.

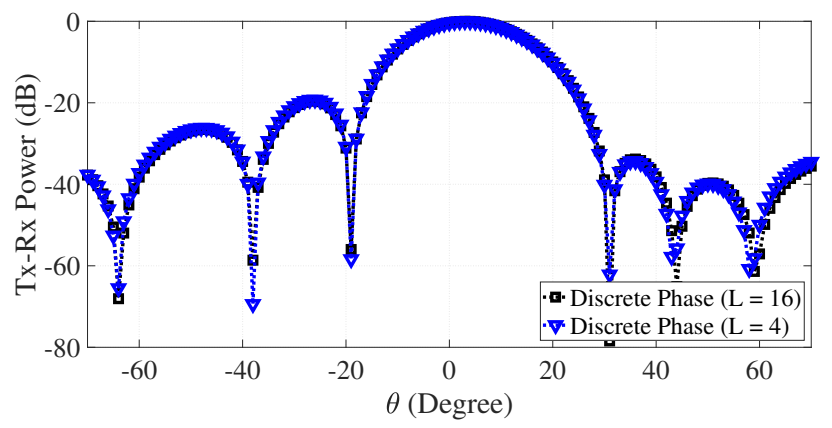

Figure 4. Transceiver pattern of the proposed algorithm, $N_{T}=$ $N_{R}=4, \theta_{0}=0, \mathrm{SNR}=20 \mathrm{~dB}, \theta_{j}=-19^{\circ}, 31^{\circ}, 44^{\circ},-38^{\circ}, 59^{\circ}$, $-64^{\circ}, \mathrm{INR}=30 \mathrm{~dB}, N=16$ and $L=4,16$.

sequences, when $L=4,16$ is plotted in Figure 4. This figure shows that the designed $M$-ary sequences are able to make a null in the direction of the signal dependent interferences.

\section{Conclusions}

In this paper, an attractive FFT-based approach is proposed to joint design of receive filter and transmit sequence with $M$-ary PSK constraint. To tackle the optimization problem, the optimum filter obtained based on the MVDR in the first step. Then, using the CD framework, the optimal code entry obtained with discrete phase constraint and using the FFT. Using the alternative optimization procedure, the code sequence and the receive filter were sequentially optimized. Our future work is to tackle the similar problem with the constraints on the waveform ambiguity function, i. e., joint design of receive filter and Doppler robust sequences.

\section{Acknowledgment}

This work was supported by FNR (Luxembourg) through the BRIDGES project "Adaptive mm-Wave 
Radar platform for Enhanced Situational Awareness: Design and Implementation (AWARDS)", CPPP17/IS/11827256/AWARDS.

\section{References}

[1] J. Li and P. Stoica, MIMO Radar Diversity Means Superiority, pp. 594-. Wiley-IEEE Press, 2009.

[2] J. A. Scheer, "Coherent radar system performance estimation," in IEEE International Conference on Radar, pp. 125128, May 1990.

[3] P. Stoica, J. Li, and M. Xue, "Transmit codes and receive filters for pulse compression radar systems," in IEEE International Conference on Acoustics, Speech and Signal Processing (ICASSP), pp. 3649-3652, March 2008.

[4] D. R. Fuhrmann and G. S. Antonio, "Transmit beamforming for MIMO radar systems using signal cross-correlation," IEEE Transactions on Aerospace and Electronic Systems, vol. 44, pp. 171-186, January 2008.

[5] A. De Maio, S. De Nicola, Y. Huang, S. Zhang, and A. Farina, "Code design to optimize radar detection performance under accuracy and similarity constraints," IEEE Transactions on Signal Processing, vol. 56, pp. 5618-5629, Nov 2008.

[6] A. Hassanien and S. A. Vorobyov, "Transmit/receive beamforming for mimo radar with colocated antennas," in 2009 IEEE International Conference on Acoustics, Speech and Signal Processing, pp. 2089-2092, April 2009.

[7] A. De Maio, S. De Nicola, Y. Huang, D. Palomar, S. Zhang, and A. Farina, "Code design for radar STAP via optimization theory," IEEE Transactions on Signal Processing, vol. 58, pp. 679-694, Feb 2010.

[8] A. Aubry, A. DeMaio, A. Farina, and M. Wicks, "Knowledgeaided (potentially cognitive) transmit signal and receive filter design in signal-dependent clutter," IEEE Transactions on Aerospace and Electronic Systems, vol. 49, pp. 93-117, Jan 2013.

[9] J. Liu, H. Li, and B. Himed, "Joint optimization of transmit and receive beamforming in active arrays," IEEE Signal Processing Letters, vol. 21, pp. 39-42, Jan 2014.

[10] S. Ahmed and M. Alouini, "MIMO-radar waveform covariance matrix for high SINR and low side-lobe levels," IEEE Transactions on Signal Processing, vol. 62, pp. 2056-2065, April 2014

[11] S. Imani and S. A. Ghorashi, "Transmit signal and receive filter design in co-located MIMO radar using a transmit weighting matrix," IEEE Signal Processing Letters, vol. 22, pp. 1521-1524, Oct 2015.

[12] A. Aubry, A. D. Maio, and M. M. Naghsh, "Optimizing radar waveform and doppler filter bank via generalized fractional programming," IEEE Journal of Selected Topics in Signal Processing, vol. 9, pp. 1387-1399, Dec 2015.

[13] S. Imani, M. M. Nayebi, and S. A. Ghorashi, "Transmit signal design in colocated MIMO radar without covariance matrix optimization," IEEE Transactions on Aerospace and Electronic Systems, vol. 53, pp. 2178-2186, Oct 2017.

[14] W. Xiong, M. Greco, F. Gini, G. Zhang, and Z. Peng, "Toeplitz matrix-based transmit covariance matrix of colocated mimo radar waveforms for sinr maximization," in 2018 IEEE International Conference on Acoustics, Speech and Signal Processing (ICASSP), pp. 3295-3299, April 2018.

[15] S. Imani, M. M. Nayebi, and S. A. Ghorashi, "Colocated MIMO radar SINR maximization under ISL and PSL constraints," IEEE Signal Processing Letters, vol. 25, pp. 422426, March 2018.
[16] J. Qian, M. Lops, L. Zheng, X. Wang, and Z. He, "Joint system design for coexistence of mimo radar and mimo communication," IEEE Transactions on Signal Processing, vol. 66 , pp. 3504-3519, July 2018.

[17] A. Hassanien and S. A. Vorobyov, "Phased-MIMO radar: A tradeoff between phased-array and MIMO radars," IEEE Transactions on Signal Processing, vol. 58, pp. 3137-3151, June 2010.

[18] P. Stoica, J. Li, and Y. Xie, "On probing signal design for MIMO radar," IEEE Transactions on Signal Processing, vol. 55, pp. 4151-4161, Aug 2007.

[19] S. Ahmed, J. S. Thompson, Y. R. Petillot, and B. Mulgrew, "Finite alphabet constant-envelope waveform design for MIMO radar," IEEE Transactions on Signal Processing, vol. 59, pp. 5326-5337, Nov 2011.

[20] Y.-C. Wang, X. Wang, H. Liu, and Z.-Q. Luo, "On the design of constant modulus probing signals for MIMO radar," IEEE Transactions on Signal Processing, vol. 60, pp. 4432-4438, Aug 2012.

[21] S. Jardak, S. Ahmed, and M. Alouini, "Generation of correlated finite alphabet waveforms using gaussian random variables," IEEE Transactions on Signal Processing, vol. 62, pp. 4587-4596, Sept 2014.

[22] M. Haghnegahdar, S. Imani, S. A. Ghorashi, and E. Mehrshahi, "SINR enhancement in colocated mimo radar using transmit covariance matrix optimization," IEEE Signal Processing Letters, vol. 24, pp. 339-343, March 2017.

[23] M. M. Naghsh, M. Modarres-Hashemi, M. Alaee-Kerahroodi, and E. H. M. Alian, "An information theoretic approach to robust constrained code design for MIMO radars," IEEE Transactions on Signal Processing, vol. 65, pp. 3647-3661, July 2017.

[24] M. Alaee-Kerahroodi, A. Aubry, A. De Maio, M. M. Naghsh, and M. Modarres-Hashemi, "A coordinate-descent framework to design low PSL/ISL sequences," IEEE Transactions on Signal Processing, vol. 65, pp. 5942-5956, Nov 2017.

[25] M. Alaee-Kerahroodi, A. Aubry, A. De Maio, M. M. Naghsh, and M. Modarres-Hashemi, "Design of binary sequences with low PSL/ISL," in 2017 25th European Signal Processing Conference (EUSIPCO), pp. 2211-2215, Aug 2017.

[26] M. Skolnik, Radar Handbook, Third Edition. Electronics electrical engineering, McGraw-Hill Education, 2008.

[27] J. Capon, "High-resolution frequency-wavenumber spectrum analysis," Proceedings of the IEEE, vol. 57, pp. 1408-1418, Aug 1969. 